\title{
Level Set Sub Pixel Edge Extraction with Corner Constrained
}

\author{
Geng Lei ${ }^{1, a}$, Liu Wen-chao ${ }^{1, b}$, Xiao Zhi-tao ${ }^{1, c,{ }^{*}}$ and Ye Kun ${ }^{1, d}$
}

${ }^{1}$ School of Electronics and Information Engineering, Tianjin Polytechnic University, Tianjin 300387, China

agenglei@tjpu.edu.cn, bqingfeng_chao@163.com, cxiaozhitao@tjpu.edu.cn, d1029627723@qq.com

Keyword: Sub pixel; Edge extraction; Corner constraint; Level set.

\begin{abstract}
In view of the problem that the traditional sub pixel edge extraction method is obviously deviated from the real edge near the corner. A new algorithm is proposed. In this paper, the corner protection function is added to the level set energy functional, which makes that the evolution of level set function is restricted at the corner, therefore this algorithm can improve the accuracy of edge extraction near the corner. The sub pixel edge extraction results of simulated and real images show that the proposed method is more accurate at the edge of the corner.
\end{abstract}

\section{Introduction}

Vision measurement technology has the advantages of non-contact, high precision and automation. And it has become the main development direction of industrial measurement technology. In the vision measurement system, the precision of edge location will directly affect the accuracy of the measurement results. And the accuracy of pixel level edge positioning is difficult to meet the actual measurement requirements. Therefore, the sub pixel edge extraction method has become one of the key techniques in the study of vision measurement technology.

At present, there are mainly three kinds of sub pixel edge detection methods, such as interpolation method, fitting method and moment method. Lv et al ${ }^{[1]}$ combined the improved Sobel algorithm and polynomial interpolation method for extracting sub pixel edge of the black sheet. The speed of the algorithm is fast. But the edge is positioned in the discrete domain. The original discrete features are preserved, especially in smooth regions, which will produce sawtooth effect. So it needs further smoothed to eliminate sawtooth. The fitting method is better, such as $\mathrm{Li}_{\text {et }} \mathrm{al}^{[2]}$ proposed elliptical sub pixel edge extraction method based on the principle of least squares; $\mathrm{Yu}^{[3]}$ proposed a one-dimensional image edge detection method based on sigmoidal function of the least squares fitting. However, these methods require that the edge point distribution of the target must be consistent with the assumed function. The different edges of the practical application are difficult to meet the above requirements. Qiu et $\mathrm{al}^{[4]}$ proposed a sub pixel edge detection algorithm based on moment and error correction table. This algorithm can reduce principle error of two-dimensional model in moment method, so as to improve the accuracy of sub pixel edge detection. The moment method based on the integral principle has well anti noise ability. But it needs local moment information to construct the edge of the linear equation, and local information of the adjacent independent leads to extract the edge discontinuities.

By the above analysis, it is known that the commonly used sub pixel edge extraction method will result in sawtooth effect. And the local model will bring the problem of edge discontinuities. In order to extract smooth, continuous sub pixel edge, Yi et al ${ }^{[5]}$ proposed level set sub pixel edge detection method. This method firstly used improved the level of diffusion method to eliminate the jagged effect of the image edge. Then it extracted the smooth and continuous sub pixel edge by using Canny operator and bicubic interpolation. Due to sub pixel edge extraction algorithms are dependent on the gray value distribution. So near the edge of the linear, gray value distribution principle is gradual change, it is easy to extract accurate sub pixel edge. But near the corner the gray value distribution is not regular, which led to the edge of topology changes. And sub pixel edge extraction results will significantly deviate from the true edge. In view of the above problems, it proposes level set sub pixels extraction method with corner constraint.

This paper first introduces CV level set model, LBF level set model and the corner detection 
algorithm based on the scatter matrix. Then it constructs level set energy functional with corner constraint. Finally it verifies the feasibility of proposed method at simulated images and real images.

\section{Related Background}

CV Model. CV model was proposed by Chan and Vese ${ }^{[6]}$. The method assumes that target and the background of the image have a large difference in gray level. Based on global average gray information, the segmentation is achieved by the establishment of the following energy function, that is:

$$
\varepsilon^{\mathrm{CV}}\left(c_{1}, c_{2}, \phi\right)=\lambda_{1} \iint_{\Omega}\left|I-c_{1}\right|^{2} H(\phi) d x d y+\lambda_{2} \iint_{\Omega}\left|I-c_{2}\right|^{2}(1-H(\phi)) d x d y
$$

$I$ is the image be segmented. $\Omega$ is the area of the image. $\phi$ is the function of the level set. $c_{1}$ and $c_{2}$ are the internal and external average gray value of the evolution curve. $\delta$ and $H$ are Dirac and Heaviside function. $\mu \geq 0, \lambda_{1}>0, \lambda_{2}>0$ are the weight coefficient.

The information of the CV model is a global quantity. It can be able to split the target and the background of the image. The speed of image segmentation is fast. But it does not contain any local information of the image. Therefore, the uneven gray level image cannot be segmented.

LBF Model. To solve the problem of CV model for non-uniform gray image segmentation, $\mathrm{Li}^{\text {[7] }}$ proposed LBF method, which introduced a Gaussian function as the kernel function of the second fitting energy function instead of CV model global binary fitting energy functional. For any $x$, the energy function of the model is defined as:

$$
\begin{aligned}
\varepsilon_{x}^{\mathrm{LBF}}\left(f_{1}, f_{2}, \phi\right)= & \lambda_{1} \int K_{\sigma}(x-y)\left|I(y)-f_{1}(x)\right|^{2} \times H(\phi(y)) d y \\
& +\lambda_{2} \int K_{\sigma}(x-y)\left|I(y)-f_{2}(x)\right|^{2} \times(1-H(\phi(y))) d y
\end{aligned}
$$

$\phi$ is the function of level set. $H$ is Heaviside function. $K_{\sigma}$ is the Gaussian function. $f_{1}$ and $f_{2}$ is local Gauss fitting value of the point $x . \lambda_{1}$ and $\lambda_{2}$ is weight coefficient. Fixed $\phi$. The $f_{1}, f_{2}$ can be got by variation method of minimizing energy function:

$$
\begin{gathered}
f_{1}=\frac{K_{\sigma}(x) *\left[H_{\varepsilon}(\phi(x)) I(x)\right]}{K_{\sigma}(x) * H_{\varepsilon}(\phi(x))} \\
f_{2}=\frac{K_{\sigma}(x) *\left[\left(1-H_{\varepsilon}(\phi(x))\right) I(x)\right]}{K_{\sigma}(x) *\left(1-H_{\varepsilon}(\phi(x))\right)}
\end{gathered}
$$

It can be seen from formula (3) and (4), $f_{i}$ is a local quantity which is average weighted gray of the local neighborhood of the image at the point $x$. Therefore, the LBF model can be used to segment the non homogenous image. But there is a new problem: because LBF does not contain any global information of the image, the segmentation results are heavily dependent on the size of the initial contour and the sensitivity to noise ${ }^{[8]}$.

Getting the Image Corner. Corner is an important feature of the image. It is very important to understanding and analyzing the image. According to the local neighborhood structure feature, Scatter matrix recognizes image and determines the angular shaped structure. Therefore, comparing with the differential operator, the method based on spread matrix is more stable and better noise resistance ${ }^{[9]}$. The corners can be got by scatter matrix, the function is showing as follows: 


$$
J_{\rho}=\left|\begin{array}{ll}
j_{11} & j_{12} \\
j_{21} & j_{22}
\end{array}\right|=\left|\begin{array}{ll}
\left(\frac{\partial I_{\sigma}}{\partial x}\right)^{2} * G_{\rho} & \left(\frac{\partial I_{\sigma}}{\partial x} \frac{\partial I_{\sigma}}{\partial y}\right) * G_{\rho} \\
\left.\frac{\partial I_{\sigma}}{\partial x} \frac{\partial I_{\sigma}}{\partial y}\right) * G_{\rho} & \left(\frac{\partial I_{\sigma}}{\partial y}\right)^{2} * G_{\rho}
\end{array}\right|
$$

$I_{\sigma}$ is the Gaussian smooth image with smooth parameter $\sigma . G_{\rho}$ is the Gaussian kernel with parameter $\rho$.

The eigenvectors $\omega^{\perp}$ and $\omega$ of $J_{\rho}$ are:

$$
\begin{aligned}
\omega & =(\cos \theta, \sin \theta)^{\mathrm{T}} \\
\omega^{\perp} & =(-\sin \theta, \cos \theta)^{\mathrm{T}}
\end{aligned}
$$

$\omega$ is the gradient direction of the image. That is the vertical direction of the image feature. $\omega^{\perp}$ is the direction of the image feature. $\theta$ is the angle between $\omega$ and horizontal axis, which can be derived from the scatter matrix:

$$
\theta=\frac{1}{2} \arctan \frac{2 j_{12}}{j_{11}-j_{22}}
$$

The local neighborhood can identify the angular structure, the calculation formula is:

$$
c_{u}=\left|\left(\nabla\left(\omega^{\perp} \omega^{\perp \mathrm{T}}\right)\right)^{\mathrm{T}} \cdot \nabla u\right|
$$

\section{The Algorithm in This Paper}

The Level Set Model with Corner Constraint. According to the analysis of CV and LBF model, the level set sub pixel edge extraction algorithm based on the advantages of CV and LBF model can avoid the influence of the initial contour and the noise on the edge extraction. Therefore, it proposed the level set sub pixel extraction method with corner constraints. It based on the level set model of combining CV and LBF and the corner neighborhood constraints.

After the combination of CV and LBF models, the energy functional of the level set is obtained as follows:

$$
E\left(\phi, c_{1}, c_{2}, f_{1}, f_{2}\right)=g \varepsilon^{\mathrm{CV}}\left(\phi, c_{1}, c_{2}\right)+\varepsilon^{\mathrm{LBF}}\left(\phi, f_{1}, f_{2}\right)+\mu R_{P}(\phi)+v L(\phi)
$$

$\varepsilon^{\mathrm{CV}}$ and $\varepsilon^{\mathrm{LBF}}$ are the energy functional of $\mathrm{CV}$ and $\mathrm{LBF}$ models. $R_{P}(\phi)$ is the regularization function of distance. $\mathrm{g}$ is the edge stopping function. $L(\phi)$ is the smoothing function. $\mu$ and $v$ are the constant terms and their values are greater than zero.

We get the corner of image based on the scatter matrix. And build the round corner protection function which the radius is $r$ on the corner, the function is as follows:

$$
\chi_{c r}(x, y)= \begin{cases}0, & (x, y) \in \operatorname{Ball}\left(\left(x_{c}, y_{c}\right), r\right) \\ 1, & (x, y) \notin \operatorname{Ball}\left(\left(x_{c}, y_{c}\right), r\right)\end{cases}
$$

After the energy level set function added corner neighborhood restrictions, it can evolve normally in flat area. But it will evolve restrictively in the corner protection area.

The level set sub pixel edge extraction energy function with corner restrictions is as follow:

$$
\begin{gathered}
E^{\prime}\left(\phi, c_{1}, c_{2}, f_{1}, f_{2}\right)= \\
\lambda_{1} \iint_{\Omega}\left|I-c_{1}\right|^{2} H(\phi) d x d y+\chi_{c r} \lambda_{2} \iint_{\Omega}\left|I-c_{2}\right|^{2}(1-H(\phi)) d x d y+ \\
\chi_{c r} \varepsilon^{\mathrm{LBF}}\left(\phi, f_{1}, f_{2}\right)+\mu \chi_{c r} R_{P}(\phi)+v \chi_{c r} L(\phi)
\end{gathered}
$$

The PDE evolution equation of the new model which is obtained by using the variation method is as follow: 


$$
\begin{gathered}
\frac{\partial \phi}{\partial t}=\delta_{\varepsilon}(\phi)\left(-\lambda_{1}\left(I-c_{1}\right)^{2}+\chi_{\mathrm{cr}} \lambda_{2}\left(I-c_{2}\right)^{2}\right)+\chi_{c r} \delta_{\varepsilon}(\phi)\left(\lambda_{1} e_{1}-\lambda_{2} e_{2}\right)+ \\
\mu \chi_{c r} \operatorname{div}\left(d_{p}(\nabla \phi) \nabla \phi\right)+v \chi_{c r} \delta_{\varepsilon}(\phi) \operatorname{div}\left(\frac{\nabla \phi}{|\nabla \phi|}\right)
\end{gathered}
$$

In the above equation:

$$
\begin{aligned}
& e_{1}(x)=\int_{\Omega} K_{\sigma}(y-x)\left|I(x)-f_{1}(y)\right|^{2} d y \\
& e_{2}(x)=\int_{\Omega} K_{\sigma}(y-x)\left|I(x)-f_{2}(y)\right|^{2} d y \\
& \left\{\begin{array}{c}
c_{1}=\frac{\int_{\Omega} I H_{\varepsilon}(\phi) d x d y}{\int_{\Omega} H_{\varepsilon}(\phi) d x d y} \\
c_{2}=\frac{\int_{\Omega} I\left(1-H_{\varepsilon}(\phi)\right) d x d y}{\int_{\Omega}\left(1-H_{\varepsilon}(\phi)\right) d x d y}
\end{array}\right.
\end{aligned}
$$

The Algorithm Implementation. The algorithm process is as follow:

(1) The result of binarization processing of the original image is as the initial level set function. It can be defined as the follow:

$$
\phi_{0}(x)=\left\{\begin{array}{cc}
2 & x \in \Omega_{0} \\
-2 & x \in \Omega \backslash \Omega_{0}
\end{array}\right.
$$

(2) According to the formula (8) for the image corner, and by the formula (10) for the term of corner protection.

(3) According to the formula (13), (14), (15), the values of $e_{1}, e_{2}, c_{1}, c_{2}$ are calculated. When we bring them into the equation (12), the $\partial \phi / \partial t$ will be calculated. The level set function $\phi_{n+1}=\phi_{n}+\Delta t \cdot(\partial \phi / \partial t)$ will be updated at the same time.

(4) Check whether the counts of the level set evolution is up to the preset number of times. If the number of times does not meet the conditions, the steps (3) will be continued. If meet the number of times, the algorithm will be terminated.

\section{Experimental Results and Analysis}

The experimental parameters are selected as the binarization threshold 0.7 . The iteration step size $\Delta t=0.1$. The weight coefficient $\lambda_{1}=\lambda_{2}=1$. The number of iterations $n=10$.

The Experiment Results of Simulated Images. Constructed a standard rectangular image, the image size is $128 \times 128$ pixels, as shown in Fig. $1(\mathrm{a})$. The distance from the left of the image to the vertical line is 30 pixels. To the horizontal straight line is 30 pixels, and the length of the vertical straight line and the horizontal straight line are 68 pixels. The standard rectangle image is smoothed by $3 \times 3$ neighborhood to generate the test image (Fig. 1(b)). Fig. 1(c) (the magnification image of the red rectangle area of Fig. 1(b)) is the edge extraction result. The green line is real edge. Green points are the detected corner points. The Fig. 1(c) show that the algorithm of canny moment sub pixel (blue) and the algorithm of this paper (red) have little difference on the flat area. But the algorithm of canny moment sub pixel extracting the edge of the inflection points in the vicinity are deviation from the real edge points. Due to the limits of the corner protection function, the algorithm of level set with corner constraint is more close to the true edge. The algorithm of this paper has better edge extraction effect. 


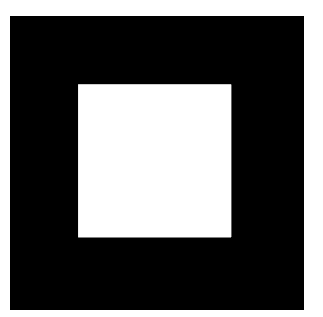

(a) Sorce image

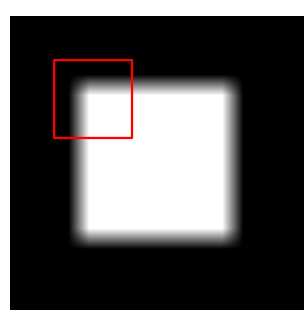

(b) Test image

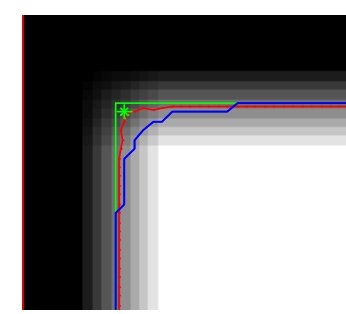

(c) Edge extraction image

Fig. 1 Right angle edge extraction result

Then, the experimental image with the triangle size $300 \times 300$ pixels is constructed, as shown in Fig. 2(a). After $3 \times 3$ neighborhood smoothing generating experimental images used in the experiment (Fig. 2(b)), the edge extraction is performed using the canny moment sub pixel algorithm and the level set method. Extraction results are shown in Fig. 2(c) and Fig. 2(d) (Fig. 2(c) and Fig. 2(d). It is a local magnification image in the red square region of Fig. 2(b)). From Fig. 2(c) at the acute angle edge detection results show that the canny moment extraction algorithm generates the fracture phenomenon, the algorithm of this paper has the better continuity. But after smoothing of the acute angle, the gray value near the corner is averaging that leads to gray values were almost the same. The level set method with corner constraint in acute office although did not reach the real point. But the effect of edge extraction has been improved. The Fig. 2(d) at an obtuse angle edge extraction results show that the canny moment sub pixel extraction results deviate from the true edge and the algorithm of this paper due to the corner constraint is more close to the real edge.

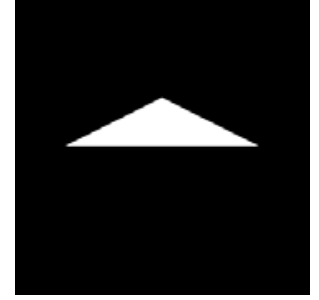

(a) Original image

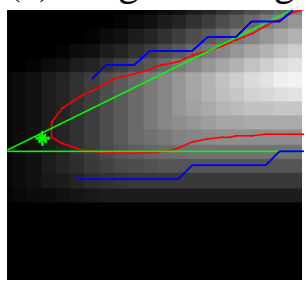

(c) Acute angle result

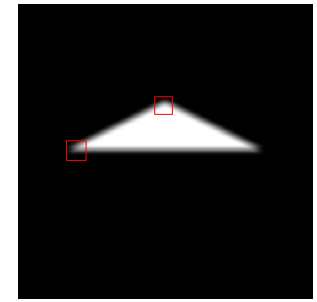

(b) Test image

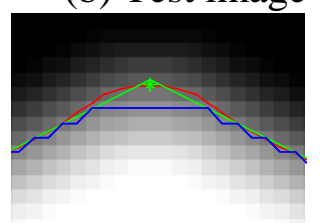

(d) Obtuse angle result

Fig. 2 The extraction results of triangle edge

Real Image Experiment. Using Canny moment sub pixel method, threshold sub pixel method and level set sub pixel method with corner constraint for sub pixel edge extraction. Then compare the effects of edge extraction of planar images collected by the measurement system. Fig. 3(a) is the plane parts of the original. Fig. 3(b) is the right angle edge in the image extraction results. It can be seen that due to the reason of corner gray value distribution. Canny moment and the threshold of the sub pixels extracted from the edge of the phenomenon of contraction. However, the level set method with corner constraint can get a very well corner edge extraction effect. Fig. 3 (c) is an acute angle extraction results. The acute angle extraction results of Canny sub pixel (blue) appear the condition of fracture. But, this method (red) of this paper and threshold sub pixel edge extraction method (green) detect the continuous edge, and the algorithm of this paper performs better. The performs of the obtuse angle edge extraction show that level set method with corner constraint (red) performs better than the other two methods (Fig. 3(d)). Fig. 3(e), Fig. 3(f), Fig. 3(g) are the result of circle, straight line. They are extraction of sub pixel edge extraction results of the three methods. It shows from the results that the three methods coincide, the extraction effect is basically the same. To sum up, the level set method with corner constraint can be more accurate to extract the edge near the corner. 


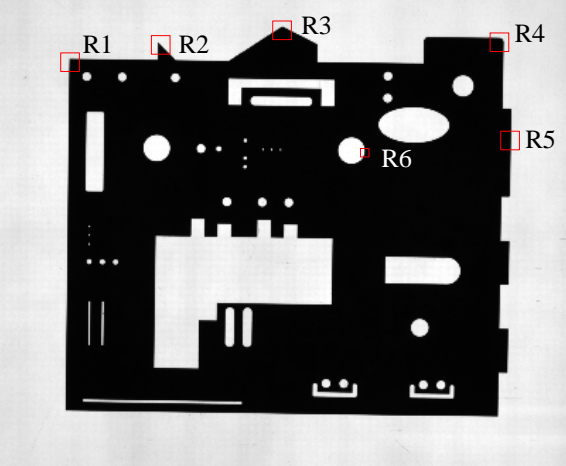

(a) The original plane parts

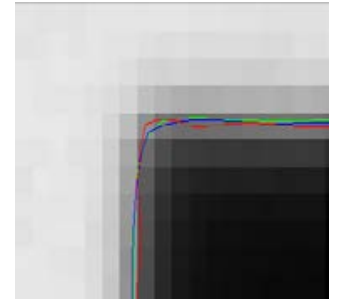

(b) The region of $\mathrm{R} 1$

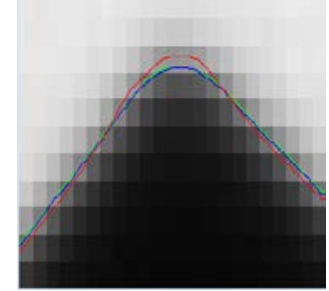

(d) The region of R3

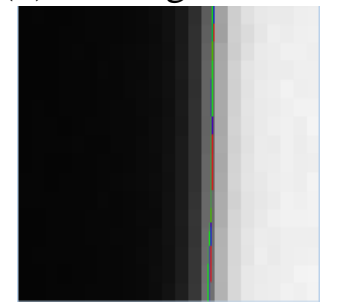

(f) The region of R5

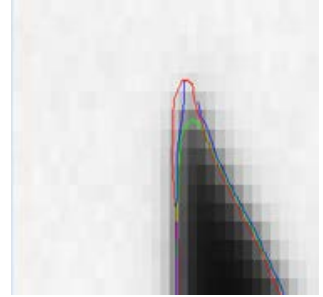

(c) The region of $\mathrm{R} 2$

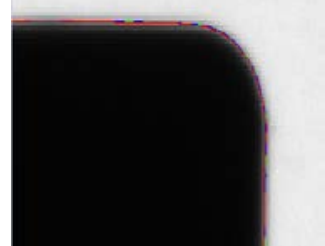

(e) The region of $\mathrm{R} 4$

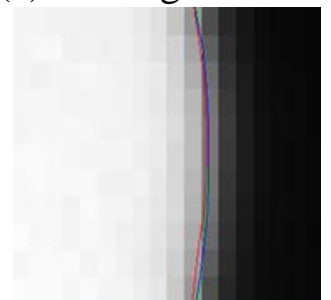

(g) The region of R6

(g) planar parts

\section{Conclusion}

Corner protection function was added in level set energy functional. So that the evolution of the level set function subject to restrictions near the corner. Thereby it can improve the accuracy of the edge extraction. The edge extraction results of simulated and real images show that proposed algorithm can improves the edge extraction accuracy near the corner. The proposed method has certain reference value for precision dimensional measurement and edge detection in practical application. In the study, we also found that edge detection accuracy of the level set model with corner constraint is mainly influenced by corner positioning accuracy. In future studies we can improve extraction accuracy near the corner by increasing corner positioning accuracy.

\section{Acknowledgements}

This paper was supported by the key technologies R \& D program of Tianjin under grant No. 14ZCZDGX00033. 


\section{REFERENCES}

[1] Lv Chun-feng, Ren Quan-hui. The silicon sheet steel image sub-pixel edge detection algorithm research based on sobel. Manufacturing Automation. Vol. 35(2013) No. 2, p. 34-36.

[2] Li Bao-zhang, Cui Yan-ping. Study on subpixel extraction of ellipse in computer vision measurement. Machinery Design \& Manufacture. (2011) No. 8, p. 142-144.

[3] Yu Hao, Liu Bing-qi, Ying Jia-ju, et al. One-dimension image edge detection method based on sigmoidal function fitting. Infrared Technology. (2014) No. 10, p. 816-819.

[4] Qiu Yun-chun, Fan Yong, Xiao De-cheng. A new error correction algorithm for sub-pixel edge detection. Computer Engineering. (2014) No. 10, p. 175-180.

[5] Yi Mo, Liu Zhong-xuan, Peng Si-long. Subpixel edge location using improved LSR, Journal of Image and Graphics. Vol. 10(2005) No.10, p. 1314-1319.

[6] Guo Jing, Jiang Jie, Cao Shi-xiang. Automatic building segmentation from remote sensing images using multi-layer level set framework. Infrared and Laser Engineering. (2014) No.4, p. 1332-1337.

[7] Li C, Kao C Y, Gore J C, et al. Implicit active contours driven by local binary fitting energy. IEEE Conference on Computer Vision \& Pattern Recognition. Minneapolis, 2007, p. 1-7.

[8] Qi Shi-le, Wang Mei-qing. “Two-stage” active contour model driven by local and global information. Journal of Image and Graphics. Vol. 19(2014) No.3, p. 421-427.

[9] Shao Wen-ze, Wei Zhi-hui. Edge-and-corner preserving regularization for image interpolation and reconstruction. Image and Vision Computing. Vol. 26(2008) No.12, p. 1591-1606. 